\title{
Inflammatory Parameters as Available Prognostic Factors for Pancreatic Cancer after Chemoradiotherapy
}

\author{
Chang Min Cho \\ Department of Internal Medicine, Kyungpook National University Chilgok Hospital, School of Medicine, Kyungpook National University,
} Daegu, Korea

\begin{abstract}
See "The Neutrophil-Lymphocyte Ratio and Platelet-Lymphocyte Ratio Are Prognostic Factors in Patients with Locally Ad-
\end{abstract} vanced Pancreatic Cancer Treated with Chemoradiotherapy” by Byung Min Lee, et al. on page 342, Vol. 12. No. 3, 2018

Pancreatic cancer is one of the worst tumors, and is the fourth leading cause of cancer-related deaths worldwide, with an overall 5-year survival rate of less than 5\%. Since nearly four decades, the survival rates have not improved substantially. Only $10 \%$ to $20 \%$ of individuals present with the disease in a potentially operable stage, and despite resection with curative intent, the median survival remains between 12 and 18 months. ${ }^{2,3}$ Although great efforts have been made in the early diagnosis and treatment of pancreatic cancer, the prognostic outcomes of pancreatic cancer remain disappointing. There are several reasons for poor prognosis: (1) a highly invasive tumor, which usually shows metastases at diagnosis; (2) relatively resistant to chemotherapy, radiotherapy, and targeted therapy; (3) harboring multiple genetic and epigenetic alterations; and (4) extremely complicated tumor-microenvironment due to close interaction with host immune system. ${ }^{4}$ Several studies focusing on cancer-related response to immunity and inflammation may provide some suggestions on the possible explanation for poor prognosis. ${ }^{5}$ Inflammation, as a major feature of tumors, has been proven to play a crucial role in the progression of malignancies, including proliferation, angiogenesis, metastasis, and chemotherapy-resistance. A variety of prognostic scores based on systemic inflammation have been proposed in the last decade.

Previous studies have demonstrated the association of systemic inflammatory response (SIR) with adverse outcomes in a variety of solid organ malignancies, including pancreatic cancer. ${ }^{6}$ It has been proven that the levels of neutrophils, lymphocytes and platelets in peripheral venous blood could be affected by tumor-induced SIR. While neutrophils function as tumor-promoting factors via the signaling pathway triggered by inflammatory cytokines, platelets also play a crucial role in the proliferation and metastasis of tumors by promoting angiogenesis, extracellular matrix degradation, and the release of growth factors and adhesion molecules. Therefore, quantitative hematological parameters have been analyzed as prognostic markers in various tumors, including pancreatic cancer.

In this issue of Gut and Liver, Lee et al. ${ }^{7}$ published a report on their study that evaluated the value of the neutrophil-tolymphocyte ratio (NLR) and platelet-to-lymphocyte ratio (PLR) as prognostic factors in pancreatic cancer. The authors divided the patients into groups based on receiver-operating characteristic values for NLR and median value for PLR of 1.89 and 149, respectively. The study identified that patients with both high NLR and high PLR revealed the worst overall survival (OS) and progression-free survival (PFS) rates compared with those with both low NLR and low PLR. The authors highlighted that elevated levels of pretreatment NLR and PLR independently and in combination significantly predicted poor OS and PFS in patients with locally advanced pancreatic cancer after chemoradiotherapy. Previous studies have proven that elevated levels of NLR and PLR correlate with unfavorable prognosis in patients who undergo curative resection for localized tumors or systemic chemotherapy for advanced tumors. ${ }^{8}$ Recently, a meta-analysis including 37 papers containing 43 cohort studies on pancreatic cancer revealed that patients with low NLR might have longer OS when compared to patients with high NLR. ${ }^{9}$ Additionally, low NLR was significantly associated with longer disease-free

Correspondence to: Chang Min Cho

Department of Internal Medicine, Kyungpook National University Chilgok Hospital, School of Medicine, Kyungpook National University, 807 Hoguk-ro, Buk-gu, Daegu 41404, Korea

Tel: +82-53-200-2608, Fax: +82-53-200-2028, E-mail: cmcho@knu.ac.kr

pISSN 1976-2283 eISSN 2005-1212 https://doi.org/10.5009/gnl18113

@ This is an Open Access article distributed under the terms of the Creative Commons Attribution Non-Commercial License (http://creativecommons.org/licenses/by-nc/4.0) which permits unrestricted non-commercial use, distribution, and reproduction in any medium, provided the original work is properly cited. 
survival and smaller tumor sizes, better differentiation, earlier stage, and low CA19-9 levels. In a study on PLR, a meta-analysis including 14 retrospective cohort studies involving 2,260 patients with pancreatic cancer showed that a high pretreatment PLR is a better predictor of shorter OS than a low PLR is. ${ }^{10}$

NLR and PLR show promise for the prediction of prognosis and response to treatment of cancers, and they are easily calculated, reproducible, and inexpensive. However, as mentioned in this article, there are several issues that should be addressed to consider NLR and PLR as prognostic factors. First, there is no consensus on the cutoff values of NLR and PLR, and thus, various cutoff values have been used in previous studies; while the receiver-operating characteristic curve was used to determine the cutoff value in some studies, median values were used in other studies. Second, most previous studies retrospectively enrolled patients with different stages and a variety of treatment modalities were applied. The heterogeneity of the study group and cutoff values may be attributed to the prognosis of patients with cancer. Finally, the mechanisms responsible for the association between NLR and PLR and poor outcomes of patients with cancer are not entirely understood. Additionally, variable factors such as infection and several host factors might affect the levels of inflammatory parameters.

Although the study by Lee et al. ${ }^{7}$ provides us with important data that will help guide us on specific topics, physicians require further studies regarding the validation of the usefulness and effectiveness of SIR markers in patients with pancreatic cancer who will undergo surgical resection or palliative management.

\section{CONFLICTS OF INTEREST}

No potential conflict of interest relevant to this article was reported.

\section{REFERENCES}

1. Siegel RL, Miller KD, Jemal A. Cancer statistics, 2017. CA Cancer J Clin 2017;67:7-30.

2. Alexakis N, Halloran C, Raraty M, Ghaneh P, Sutton R, Neoptolemos JP. Current standards of surgery for pancreatic cancer. Br J Surg 2004;91:1410-1427.

3. James TA, Sheldon DG, Rajput A, et al. Risk factors associated with earlier age of onset in familial pancreatic carcinoma. Cancer 2004;101:2722-2726.

4. Kamisawa T, Wood LD, Itoi T, Takaori K. Pancreatic cancer. Lancet 2016;388:73-85.

5. Balkwill FR, Mantovani A. Cancer-related inflammation: common themes and therapeutic opportunities. Semin Cancer Biol 2012;22:33-40

6. McMillan DC. The systemic inflammation-based Glasgow Prognostic Score: a decade of experience in patients with cancer. Cancer Treat Rev 2013;39:534-540.

7. Lee BM, Chung SY, Chang JS, Lee KJ, Seong J. The neutrophillymphocyte ratio and platelet-lymphocyte ratio are prognostic factors in patients with locally advanced pancreatic cancer treated with chemoradiotherapy. Gut Liver 2018;12:342-352.

8. Templeton AJ, McNamara MG, Šeruga B, et al. Prognostic role of neutrophil-to-lymphocyte ratio in solid tumors: a systematic review and meta-analysis. J Natl Cancer Inst 2014;106:dju124.

9. Zhou Y, Wei Q, Fan J, Cheng S, Ding W, Hua Z. Prognostic role of the neutrophil-to-lymphocyte ratio in pancreatic cancer: a metaanalysis containing 8252 patients. Clin Chim Acta 2018;479:181189.

10. Xu ZS, Zhang FP, Zhang Y, et al. Prognostic role of the pretreatment platelet-lymphocyte ratio in pancreatic cancer: a metaanalysis. Oncotarget 2017;8:99003-99012. 